\title{
Market Based Intraday Coordination of Electric and Natural Gas System Operation
}

\author{
Aleksandr Rudkevich \\ Newton Energy Group \\ arudkevich@negll.com \\ Evgeniy Goldis \\ Newton Energy Group \\ jgold@negll.com \\ Scott Backhaus \\ Los Alamos National Laboratory \\ azlotnik@lanl.gov
}

\author{
Anatoly Zlotnik \\ Los Alamos National Laboratory \\ azlotnik@lanl.gov \\ Richard D. Tabors \\ Tabors Caramanis Rudkevich \\ rtabors@tcr-us.com \\ Michael Caramanis \\ Boston University \\ mcaraman@bu.edu \\ Russ Philbrick \\ Polaris Systems Optimization \\ russ.philbrick@psopt.com
}

\author{
Pablo Ruiz \\ Boston University \\ paruiz@bu.edu \\ Richard Hornby \\ Tabors Caramanis Rudkevich \\ rhornby@tcr-us.com
}

\author{
Aleksandr Beylin \\ Newton Energy Group \\ abeylin@negll.com
}

\begin{abstract}
This paper outlines the design of an intraday market-based mechanism for coordinated scheduling of gas-fired electric generation, intra-day natural gas purchases, sales and deliveries, and underlying pipeline operation. The mechanism is based on an exchange of physical and pricing data between participants in each market, with price formation in both markets being fully consistent with the physics of energy flow. In organized nodal electricity markets, prices are consistent with the physical flow of electric energy in the power grid because the economic optimization used to clear the market accounts for the physics of power flows. In the gas system, the proposed physical operation and pricing will be based on the transient optimization approach that accounts for physical and engineering factors of pipeline hydraulics and compressor station operations. The paper provides theoretical foundations for the market mechanism.
\end{abstract}

\section{Introduction}

The growing reliance of the bulk electric power system on gas-fired generation increased the need to improve the coordination between wholesale natural gas and electricity markets.
Replacement of coal fired and nuclear plants with gas-fired generating capacity significantly increases the amount of natural gas used as fuel for power generation. In parallel, the variability of electric generation from wind and solar increases the variability of pipeline deliveries to gas-fired generators used to balance the electric grid. The resulting intraday and even sub-hourly swings in demand for natural gas as a fuel for electric generation create new challenges for pipeline operators, and - poses reliability risks for both gas pipelines and electric systems.

The need to better coordinate -both sectors to mitigate these risks is well recognized, and is reflected in the - Orders 787 and 809 by the Federal Energy Regulatory Commission (FERC), which regulates access to pipeline capacity [1,2]. Coordination mechanisms proposed to date are based on widening the scope of operational information exchanges between the -two sectors, and on adjusting the timing of these exchanges [3]. While these measures are helpful, a truly efficient coordination should be based on timely exchange of both physical and pricing data, with price formation in both markets being fully consistent with the physics of energy flow.

Electricity prices consistent with the physical flow of electric energy in the power grid are the outcome of economic optimization of power system operation in organized electricity markets administered by Regional Transmission Organizations (RTOs) [4, 5]. A similar optimization approach that accounts for physical and 
engineering factors of gas pipeline - and compressor station operations would lead to location- and timedependent economic value of natural gas consistent with the physics of gas flow. Such an approach have been formulated in [6] under the simplified assumption of steady state pipeline flows. A more general formulation considers a truly dynamic transient flow of natural gas

This paper relies on Ref. [8] in which a transient pipeline optimization problem that maximizes total market surplus over supply and offtake schedules has been formulated. Market Surplus in this context is defined as the sum of the producer/supplier surplus and consumer/buyer surplus. Producer surplus is derived whenever the price the producer receives exceeds the value they are willing to accept for the goods they sell. Similarly, consumer surplus is derived whenever the price the consumer ends up paying for a good is below the value they are prepared to pay. Market surplus is the sum of individual surpluses over all consumers/buyers and producers/sellers participating in the market.

The appropriate transient optimization solution dynamically allocates pipeline capacity among transactions between suppliers and consumers based on the economic value of these transactions. Compressor operations and line pack are optimized in conjunction with the selection of location-dependent offers to sell, and bids to buy, natural gas. Location-based (nodal) prices of natural gas are computed as dual variables corresponding to the nodal flow balance constraints in the optimal solution, and reflect the time- and locationdependent economic value of gas in the network. Reference [8] introduces the Locational Trade Values (LTVs) of natural gas as shadow prices of the nodal flow balance constraints. One of the key results of that paper is the proof of the revenue adequacy of the market settlement mechanism based on LTVs.

The objective of the current paper is to introduce the proposed design of the Gas Balancing Market (GBM) as a critical element for economically efficient gas-electric coordination. This coordination mechanism will facilitate the timely exchange of both physical and pricing data between participants in each market, with price formation in both markets being fully consistent with the physics of energy flow. Physical data would be intra-day (e.g., hourly) gas schedules (burn and delivery) and pricing data would be bids and offers reflecting willingness to pay and to accept. Location-based gas prices would be obtained using optimization of transient pipeline flow models. Inputs to the pipeline optimization problem include prices that power plants are willing to pay for gas, as derived from nodal electricity prices that are produced by power system optimization.

\section{Gas Balancing Market}

\subsection{Motivation}

Electric and natural gas networks follow distinct but inter-related decision processes of scheduling their operations. The timeline of these processes are schematically presented in Fig. 1. As one can see in this timeline, there exists a highly intricate succession of decision cycles on the electric side and natural gas side. A gas-fired generating unit considering to operate on the next electric day (which begins at midnight) should submit an offer to the Day-Ahead market on the prior day by 10:30 AM Eastern Time. Prior to that, the asset manager for the unit would line up gas supply and delivery. Supply will be arranged at a pipeline receipt point at a bilaterally negotiated price. Shipment of gas from the receipt point to the delivery point on the pipeline could be arranged on a firm basis through the capacity release mechanism or on a non-firm basis by obtaining interruptible capacity. This process yields a preliminary supply arrangements and gas prices. These prices, although not backed up by delivery guarantee, inform electric generators on how to bid in the day-ahead (DA) electricity market. This process exposes transacting parties to various kinds of risk.

Once the DA market clears and the financially binding operational schedules for electric generators are determined, generators have just enough time to make delivery nominations with the pipeline for the next gas day. If the nominations are confirmed in the Timely and/or Evening cycles on the gas side, daily delivery quantities are essentially guaranteed. However, even if confirmed, the quantities needed by the generator may be different from those preliminary arranged and the difference must be settled between the parties.

If deliveries needed by the generator are not confirmed due to pipeline capacity limitations, generators will face significant financial exposure as they are obligated to deliver power but have no gas to produce it. This financial exposure is two-fold: generator may need to acquire under-delivered power in the real-time market and also may be facing nonperformance penalties if the electric under-delivery occurs at the time of scarcity.

Even if the daily delivery quantity is confirmed, the pipeline typically expects that gas will be taken in equal quantities in each hour of the gas day (a ratable quantity). Generators, however, need non-ratable quantity which pipelines may be able to accommodate but cannot guarantee. 
Furthermore, most fast-start combined cycle generators and gas turbine peaking facilities are not committed in the DA market. Instead those units are typically scheduled through the hourly reliability updates or close to the real-time market. These "lastminute" decisions simply do not fit into the existing decision cycles on the gas side. For these generators which are critical for maintaining reliability of the electric service and providing essential ancillary services there is no transparent mechanism on the gas side under which they can purchase gas and schedule delivery as needed. Sudden ramps required by these generators may cause operational problems on the pipeline side. If these generators receive no gas, this will jeopardize operational reliability of the electrical grid, while delivering gas to these units may jeopardize reliability of the pipeline system.

We propose to solve these problems through the formation of the hour-by-hour natural gas balancing market that would allow market participants to trade deviations from approved ratable schedules in the Timely and Evening Cycles.

These deviations could be traded through the formal optimization based auction-type market mechanism as described in the next section. Such an auction could be run on an hourly basis using a rolling horizon approach, such that each hour the auction would optimize the system for multiple hours (e.g. 36 hours or even more). Such a balancing market would provide a repeated forward-looking price discovery mechanism to help the gas and electric sectors to efficiently coordinate their operations.

\subsection{Gas Balancing Market in Words}

The proposed Gas Balancing Market (GBM) will have voluntary participation and will be administered by a pipeline specific market administrator.

GBM will function as a two-sided auction conducted on the gas pipeline network and administered by the Market Operator. Network nodes are primarily custodial meters where gas change hands, compressor stations and pipe interconnection points. Network edges are pipes physically connecting nodes. Auction participants are buyers and sellers of gas submitting price/quantity (P/Q) offers/bids to sell/buy gas at network nodes. Their buy/sell positions are primarily driven by the need to buy gas above the ratable schedules or the desire to sell gas in excess of ratable schedules. Thus, the same market participant may act as a buyer in one hours and as a seller in another hour. Ratable schedules would be based on deliveries confirmed at the Timely or Evening nomination cycle. In addition, the market would allow participation of buyers and sellers which have no dayahead confirmed schedules or firm capacity rights.

Offers and bids are submitted with an hourly time step for a multi-hour optimization horizon (e.g., 36 hours).

Auctioneer's objective function is to maximize, over the optimization horizon, the market surplus between accepted bids and offers less compressor costs of running the pipeline.

The auction repeats periodically (e.g. every hour) as shown in Fig. 2, which also depicts the transition of this repeated auction from one gas day to another.

The optimization problem is formulated subject to the dynamic transient pipeline flow equations and must satisfy key engineering constraints. The latter are limitations on the maximum allowed operating pressure at each pipe, minimum pressure requirements at each node, horsepower limitations and compression ratios of compressors.

The outcome of the auction includes:

- Hourly schedules for receipt and deliveries of natural gas over the optimization horizon for each buyer and seller and for each node of the network. These schedules are the net results of ratable schedules and buy/sell positions cleared in the market;

- Hourly shadow prices of nodal mass balance equations referred to as Locational Trade Values (LTVs) of natural gas;

- Operational compressor setting and compression ratios for each compressor station;

- Pressure dynamics at pipes and nodes;

- Market clearing for the first hour of the optimization horizon will be ex post to actual deliveries in that hour. Market clearings for all subsequent hours of that horizon will be ex ante;

- All cleared positions for all hours of the optimization horizon will be financially binding.

These market results will be financially binding, thus giving gas buyers and sellers assurance of obtaining needed gas or liquidating an excess supply at a price that is acceptable and known ahead of the delivery. Since the market is cleared multiple times for the same period, future uncertainties are resolved. This is because the positions taken in earlier instances of the GBM could be liquidated at a later time if necessary. In sum, the proposed market structure will provide a transparent and efficient mechanism for nearly realtime gas trading and corresponding price discovery.

The proposed GBM timing is aligned with nomination cycles that are in place for natural gas pipeline in the United States. The first trading of a particular GBM cycle is aligned with the completion of the Evening Nomination Cycle (21:00 Central Time) 
and would have an optimization horizon of 36 hours which will cover the remaining portion of the current gas day (from 21:00 to 09:00 of the following day) and the entire gas day from 09:00 of the following day to 09:00 of the day after. The next trading will occur at 22:00 and will have an optimization horizon of 35 hours also ending at 09:00 of the day after the following day.

Because the optimization is conducted subject to the gas flow physics and engineering constraints, market clearing assures feasibility of delivery schedules identified.

\section{The GBM Optimization Problem}

\subsection{Pipeline Network Representation}

The GBM market clearing process is designed as a large-scale, system-wide transient optimization of a pipeline network operation. Although we employ several simplifications for the purpose of this paper, the modeling can be extended to capture more complex physical and engineering aspects. Specifically, we assume isothermal flow through a horizontal pipeline with constant gas composition, and where gas compressibility is specified using the CNGA method in the equation of state $[10,11]$. We also assume that flow changes are sufficiently slow so as not to excite waves or shocks, so that second order terms may be removed from the dynamic equations, and relatively coarse discretizations in both space and time may be used. The important parameters for a pipe are length, diameter, and the Colebrook-White friction factor. The dynamics of gas flow within the pipe can then be modeled using the isothermal Euler equations in one dimension, with the inertia and gravity terms omitted $[12,13]$.

For simplicity in this paper, compressor stations and regulator elements are modeled as two-ended flow devices that can enforce the given time-dependent pressures on a specified side, such as the discharge pressure. Theoretical power for compressors is computed as a simple function of volumetric flow rate $\phi$ and compression ratio $\alpha$, given by $|\phi|\left[\max \left(1, \alpha^{h-1}\right)-1\right]$, where $h=(\gamma-1) / \gamma$, and $\gamma$ is the specific heat capacity ratio of the gas. In this paper we do not model removal of gas from the pipeline to fuel compressor station operation, as it is a relatively small quantity of the through-flow (e.g. $0.25 \%$ ) and does not significantly affect marginal prices.

We consider a system of pipes, compressors, and regulators that are connected at nodes. Within the pipes, the mass flux and density evolve according to the simplified Euler equations. This collection of elements connected at nodes is considered as a directed graph $G=(\mathcal{V}, \mathcal{E})$, where each segment $e=\{i, j\} \in \mathcal{E}$ is an edge that connects two nodes $i$ and $j$ in the set of nodes $V$. The instantaneous state within an edge is characterized by the pressure $p_{i j}$ and flow $\phi_{i j}$, which for pipes are functions of both time on an interval $[0$, $\mathrm{T}]$ and space on an interval $\left[0, \mathrm{~L}_{\mathrm{e}}\right]$, where $\mathrm{T}$ is the optimization horizon and $\mathrm{L}_{e}$ is the length of pipe segment $e$. We assign a positive flow direction on each pipe, and then derive equations that relate the pressure and flow at the boundaries of a pipe segment to the conditions at a node. Each node is classified as either a pressure (slack) node $j \in \mathcal{V}_{P}$, where a pressure profile $p_{j}$ in time is specified and flow is a free variable, or a flow node $j \in \mathcal{V}_{F}$, where the time-dependent flow $d_{j}$ entering or leaving the network is specified and pressure is free. At least one pressure node must be included in the model so that there is a degree of freedom in flow to ensure that the initial value problem in simulations used to validate the optimization solution is well-posed. This will typically be a large source point, such as a supply interconnection or storage unit, where the pressure is a given boundary condition. An illustration and a more detailed description of the variables used in such reduced nodal modeling are illustrated in Figure 3. Each node must satisfy the Kirchhoff-Neumann flow balance condition that requires mass moving through the node to be conserved. This stipulates that the sum of incoming flows is equal to the sum of outgoing flows plus any consumption $d_{j}$ at that node. Each specified flow node $j \in \mathcal{V}_{D}$ is also assigned an internal nodal pressure, $p_{j}$ which serves as an auxiliary variable. A compressor can boost the pressure difference between pipe segments attached at its inlet and outlet nodes. This induces extra compatibility equations into the description of the coupled system of differential equations.

Numerical simulations using the referenced representation of pipeline networks are validated in [9] where simulated results are benchmarked against SCADA measurements for a segment of Tennessee pipeline.

\subsection{Optimization Problem Formulation}

We formulate an optimal control problem (OCP) subject to partial differential equation (PDE) constraints for gas pipeline networks, for which the edge dynamics and nodal conditions described above form the dynamic constraints. The aim is to maximize an economic objective function in the form of the market surplus. This market surplus is maximized in 
total over the optimization horizon $[0, T]$ which may be a 24-hour day or longer. At each point in time, market surplus is computed as the difference between the the economic value consumers (buyers) are placing on (willing to pay for) gas purchases $\hat{d}_{j}(t)$ at nodes $j$ minus the value of gas which producers (sellers) are placing on (willing to accept for) gas sales $\hat{s}_{j}(t)$ at nodes $j$. The inputs to the problem consist of the bid and offer prices $c_{j}^{d}(t)$ and $c_{j}^{s}(t)$, respectively that buyers or sellers at a node $j$ are willing to pay or accept at time $t$ within the optimization horizon $[0, T]$. In addition to price bids, quantity bids are also supplied in the form of pre-existing contracts $\bar{q}_{j}(t)$, minimum and maximum offtake curves $d_{j}^{\min }(t)$ and $d_{j}^{\max }(t)$ of buyers, and minimum and maximum supply curves $s_{j}^{\min }(t)$ and $s_{j}^{\max }(t)$ of suppliers. The economic objective is maximized subject to a collection of constraints that describe pipeline system operation, and where the control variables include compression ratios $\underline{\alpha}_{i j}(t)$ of gas compressors or compression ratios in the system. The PDE dynamics for gas flow on each pipe $(i, j)$ are enforced, as well as flow balance at each node $j$ and pressure changes caused by compression. Inequality constraints include minimum and maximum limits on pressure on each pipe, maximum power limits of each compressor, and maximum and minimum withdrawals or injections for buyers and sellers. For simplicity, we choose terminal conditions on the state and control variables to be time-periodic. Alternative initial and terminal conditions such as mass balance over the optimization period on certain subsystems could be included instead.

Crucially, we assume that no discrete changes to the network topology occur during the optimization period. Thus, no discrete variables, such as binary on/off switches, are included in the formulation. While compressor stations are in reality subject to complex operational limitations, we demonstrate that, in principle, nonlinear station constraints can be included in a computationally tractable manner as long as the modeling does not include on/off variables. For instance, a large compressor station with multiple (e.g. a dozen or more) units that receive flow from a common feeder and deliver flow to a common header can be modeled as a single theoretical boost ratio for the purpose of optimization. Modern compressor stations often have control systems that can be set to track a set point or reference signal for discharge pressure or horsepower. Thus we suppose that the management of individual units is automated, and focus on the large-scale system effects of control actions while supposing that subsystems can be taken care of at a local level. The optimization problem is stated as follows:

Maximization of market surplus

$$
\max \sum_{j \in V} \int_{0}^{T} c_{j}^{d}(t) \hat{d}_{j}(t) d t-\sum_{j \in V} \int_{0}^{T} c_{j}^{s}(t) \hat{s}_{j}(t) d t
$$

s.t.

Mass conservation

$$
\partial_{t} \rho_{i j}+\partial_{x} \phi_{i j}=0, \quad(i, j) \in E .
$$

Momentum conservation

$$
\partial_{t} \phi_{i j}+\partial_{x} \rho_{i j}=-Z\left(p_{i j}\right) R T_{w} \frac{f_{i j} \phi_{i j}\left|\phi_{i j}\right|}{2 D_{i j} p_{i j}},(i, j) \in E
$$

Equation of state

$$
p_{i j}=Z\left(p_{i j}\right) R T_{w} \rho_{i j}, \quad(i, j) \in E .
$$

Nodal flow balance

$$
\begin{aligned}
& \sum_{j \in \partial_{-} j} A_{j k} \underline{\phi}_{j k}(t)-\sum_{i \in \partial_{+} j} A_{i j} \bar{\phi}_{i j}(t)-\bar{q}_{j}(t) \\
& -\left(\hat{s}_{j}(t)-\hat{d}_{j}(t)\right)=0, \quad j \in V .
\end{aligned}
$$

Compressor boost

$$
\begin{aligned}
\underline{p}_{i j}(t) & =\underline{\alpha}_{i j}(t) p_{i}(t), \quad(i, j) \in E, \\
\bar{p}_{i j}(t) & =\bar{\alpha}_{i j}(t) p_{j}(t), \quad(i, j) \in E .
\end{aligned}
$$

Pressure limits

$$
\begin{aligned}
& p_{i j}^{\min } \leq p_{i j}(t, 0) \leq p_{i j}^{\max }, \quad(i, j) \in E, \\
& p_{i j}^{\min } \leq p_{i j}\left(t, L_{i j}\right) \leq p_{i j}^{\max }, \quad(i, j) \in E .
\end{aligned}
$$

Boost upper limits

$$
\begin{array}{ll}
\varepsilon_{i j}\left|\underline{\phi}_{i j}(t)\right|\left(\left(\underline{\alpha}_{i j}(t)\right)^{h}-1\right) \leq \underline{E}_{i j}^{\max }, & (i, j) \in E, \\
\bar{\varepsilon}_{i j}\left|\bar{\phi}_{i j}(t)\right|\left(\left(\bar{\alpha}_{i j}(t)\right)^{h}-1\right) \leq \bar{E}_{i j}^{\max }, & (i, j) \in E .
\end{array}
$$

Boost lower limits

$$
\underline{\alpha}_{i j}(t) \geq 1, \bar{\alpha}_{i j}(t) \geq 1 \quad(i, j) \in E .
$$

Supply limits

$$
s_{j}^{\min }(t) \leq \hat{s}_{j}(t) \leq s_{j}^{\max }(t) .
$$

Demand limits

$$
d_{j}^{\min }(t) \leq \hat{d}_{j}(t) \leq d_{j}^{\max }(t)
$$

Additional constraints that require the total mass of gas in the system at the end of the optimization horizon to return to the initial state may be added.

Equations (1) - (11) use the following notations:

$V$ : set of nodes

$E$ : set of pipes

$T$ : length of the optimization horizon 
$R:$ gas constant (depends on gas gravity)

$T_{w}$ :working temperature (assumed constant throughout the system)

$Z(\bullet)$ : gas compressibility as a function of pressure (working temperature)

$f_{i j}$ : Colebrook-White friction on pipe $(i, j)$

$D_{i j}:$ diameter of pipe $(i, j)$

$A_{i j}:$ cross-sectional area of pipe $(i, j)$

$L_{i j}:$ length of pipe $(i, j)$

$c_{j}^{s}$ : supply offer price at node $j$ at time $t$

$c_{j}^{d}$ :demand bid price at node $j$ at time $t$

$\hat{s}_{j}(t), \hat{d}_{j}(t)$ : variable supply and demand at node $j$ at time $t$

$\rho_{i j}(t, x):$ density on pipe $(i, j)$ at time $t$ and location $x$

$p_{i j}(t, x):$ pressure on pipe $(i, j)$ at time $t$ and location $x$

$\phi_{i j}(t, x):$ mass flux on pipe $(i, j)$ at time $t$ and location $x$

$\underline{p}_{i j}(t), \bar{p}_{i j}(t)$ : pressure at the inlet and outlet of pipe $(i, j)$ at time $t$

$\phi_{i j}(t), \bar{\phi}_{i j}(t)$ : mass flux at the inlet and outlet of pipe $(i, j)$ at time $t$

$p_{i j}^{\min }, p_{i j}^{\max }:$ minimum and maximum pressure on pipe $(i, j)$

$\varepsilon_{i j}, \bar{\varepsilon}_{i j}:$ compressor energy usage factor of compressors at the inlet and outlet of pipe $(i, j)$

$\underline{\alpha}_{i j}(t), \bar{\alpha}_{i j}(t)$ : boost ratios of compressors at the inlet and outlet of pipe $(i, j)$

$h$ : compressor energy function exponent (depends on gas specific eat capacity ratio)

$\underline{E}_{i j}^{\max }, \bar{E}_{i j}^{\max }:$ maximum energy (horsepower) of compressors at the inlet and outlet of pipe $(i, j)$

$s_{j}^{\min }(t), s_{j}^{\max }(t)$ : minimum and maximum supply from node $j$ at time $t$

$d_{j}^{\min }(t), d_{j}^{\max }(t)$ : minimum and maximum demand at node $j$ at time $t$

\section{On the Coordination Dynamics}

The coordination dynamics between the gas and electric networks could be envisioned as a periodic exchange of physical and pricing information.

Consider the end of the Evening Cycle as a point at which GBM has the longest optimization horizon which lasts through the end of the current gas day and through the next gas day (total of 36 hours). Once this cycle of the GBM clears, it yields a 36-hour long projection of LTVs. Gas-fired generators could use these LTVs to update their real-time bids to the electricity market for the next hour and to update their gas purchasing decisions for future hours in which the generator may have been scheduled to operate as an outcome of the previously cleared DA market.

While Generators can use LTVs to develop their price offers in the electric market, they can use electric LMPs to develop bids and offers in the GBM. Indeed, consider a generating unit scheduled in the DA market to operate in a future hour. Its projected gas use in that hour is greater than the daily ratable quantity (total daily gas use divided by 24) and therefore in that hour, the generator needs to purchase gas in the balancing market. The price the generator will be willing to pay for that additional gas could be as high as

$$
C_{\max }=(L M P+R-V O M) / H
$$

where $C_{\max }$ is the gas price ceiling, $L M P$ is the electric LMP at the generator's node, VOM is the non-fuel variable operating and maintenance costs of generator, and $H$ is the generator's heat rate. The term $R$ reflects an additional risk premium generators would factor into their willingness to pay for gas to avoid excess charges they may face in the real-time electricity market and potentially high nonperformance penalties during scarcity events. Additional risk factor may be needed for cycling generators at times when they operate at their minimum capacity when electricity prices that are too low to recover their operating costs.

On the other hand, if in a future hour the generator is long on gas (for example, if it is not scheduled to operate in that hour but it is still expected to take a ratable quantity of gas), it will be willing to sell that gas in the GBM. Simplistically, the price floor the generator may be willing to accept could be as low as zero, although dynamic consideration and/or opportunity/obligations to provide ancillary services may affect these pricing considerations.

Even a relatively simplistic coordination mechanism as described here will likely result in an improved overall gas-electric system efficiency. Indeed, pipeline 
congestion will be reflected in higher LTVs at certain locations. Generating units taking gas at these locations will see higher hourly prices and translate those into higher bids submitted to the electricity market. Electric System Operator could respond by reducing output of such generators and to the extent possible will be replacing them with generation with lower cost of gas or other fuels. That in turn will reduce demand for natural gas at constrained pipeline locations and therefore relieve pipeline congestion and reduce natural gas prices to other consumers.

\section{Policy Implications}

The design of the proposed GBM mechanism is particularly suitable for the regulatory structure and market design currently in place in the United States. GBM will require minimal regulatory reform and is intended to keep the existing and vibrant market structure in place in the USA intact.

As stated earlier in Section 2.2, we envision GBM as a pipeline-specific market which does not necessarily require the formation of a regional organization to coordinate the operations of several interconnected pipelines. GBM can be instituted at a pipeline level and could also be set to serve only a part of the pipeline system, to simplify an initial implementation.

We propose also that participation in the GBM be voluntary. Market participants that are satisfied with the existing operational and market mechanism are not required to submit bids or offers into the GBM.

We chose the proposed timing associated with the GBM decision cycles to minimize its impact on the operation of the current market and scheduling decision cycles. GBM will have little or no operational overlap with the existing day-ahead trading of natural gas, supply nominations and scheduling. By design, GBM is a market that is added to where none exists. These factors should reduce the hurdles for implementing the GBM within the regulatory structure of interstate pipelines regulated by FERC and possibly by intrastate pipeline in jurisdiction of state regulators,

Participation in the GBM offers benefits to market participations both on the gas side and on the electric side. On the gas side, the GBM will provide relief of pipeline constraints through LTV-sensitive optimization of compressors helping to determine the most efficient line pack strategy over time and across the system to assure higher delivery to locations with the highest value of gas. At the same time, redispatch of electric generation in response to dynamically formed LTVs will provide additional relief of pipeline constraints. In the long run, creation of the GBM will help pipeline customers make investment decisions. Gas-fired generators, especially those owned by the
Independent Power Producers, are reluctant to enter long-term contracts for Firm Transportation capacity. Generation owners are exposed to a significant financial risk of not being able to recover fixed cost costs associated with holding FT transportation rights at the time when the power plant is running. The existing capacity release mechanism and daily pricing structure do not provide sufficient price granularity to help generating companies appropriately evaluate the risk of making a decision to acquire long-term FT rights. With the GBM in place, a generating company can rely on the associated price discovery mechanism with hourly granularity to assess the value of FT rights when those are under-utilized. That risk exposure could be compared to the risk exposure on the electric side and associated with the generator nonperformance due to inability to acquire gas deliveries.

LTV-based pricing will help pipeline owners to better identify constrained system elements with better granularity. Using LTV-based pricing, pipeline owners could more precisely assess economic benefits of alternative solutions and justify investments in economic solutions before regulatory agencies.

On the electric side, gas-fired generators could rely on hourly gas trade values (LTVs) to support bidding into DA and RT markets leading to a more efficient commitment and dispatch of the electrical grid. Relying on GBM will simplify gas purchases for gasfired fast-start power plants that clear in the real-time power markets and/or that are called upon to provide ancillary services. Redispatch of electric generation will reduce electricity prices in response to high gas LTV under scarcity caused by pipeline constraints.

\section{References}

[1] "Federal Energy Regulatory Commission (FERC) order \#787," www.ferc.gov/CalendarFiles/20131115164637-

RM13-17-000.pdf.

[2] "Federal Energy Regulatory Commission (FERC) order \#809,"http://www.ferc.gov/whats-

new/commmeet/2015/041615/M-1.pdf

[3] MITEI. (2013) Growing concerns, possible solutions:

The interdependency of natural gas and electricity systems. [Online]. Available: http://mitei.mit.edu/publications/ reports-studies/growing-concerns-possible-solutions

[4] E. Litvinov, "Design and operation of the locational marginal prices-based electricity markets," Generation, Transmission Distribution, IET, vol. 4, no. 2, pp. 315-323, February 2010.

[5] Forward Market Operations, "Energy \& Ancillary Services Market Operations, M-11 Rev. 75," PJM, Tech.

Rep., 2015.

[6] A. Rudkevich, and A. Zlotnik. "Locational Marginal Pricing of Natural Gas subject to Engineering 
Constraints." Proceedings of the 50th Hawaii International Conference on System Sciences, pp. 3092-3101, 2017

[7] E. G. Read, B. J. Ring, S. R. Starkey, and W. Pepper, "An LP-based market design for natural gas," in Handbook of networks in power systems II. Springer, 2012, pp. 77-113.

[8] A. Zlotnik, A. Rudkevich, M. Vuffray, S. Misra,

"Dynamic Marginal pricing for Optimal Scheduling of Natural Gas Supply and Delivery over a Pipeline Network," In preparation

[9] A. Zlotnik, A. Rudkevich, E. Goldis, P.A. Ruiz, M. Caramanis, R. Carter, S. Backhaus, R. Tabors, R. Hornby, D. Baldwin. "Economic Optimization of Intra-Day Pipeline

Flow Schedules using Transient Flow Models." Proceedings of the PSIG 2017 Conference, Atlanta, GA, May.

[10]. E. S. Menon, Gas pipeline hydraulics. CRC Press,
[11]. A. L. Lee, M. H. Gonzalez, and B. E. Eakin. "The viscosity of natural gases." Journal of Petroleum Technology vol. 18, no. 8, pp. 997-1000, 1966

[12]. A. Zlotnik, M. Chertkov, and S. Backhaus, "Optimal control of transient flow in natural gas networks," in 54th IEEE Conference on Decision and Control, Osaka, Japan, 2015, pp. 4563-4570.

[13]. A. Osiadacz. "Simulation of transient gas flows in networks." International journal for numerical methods in fluids 4.1 (1984): 13-24. 


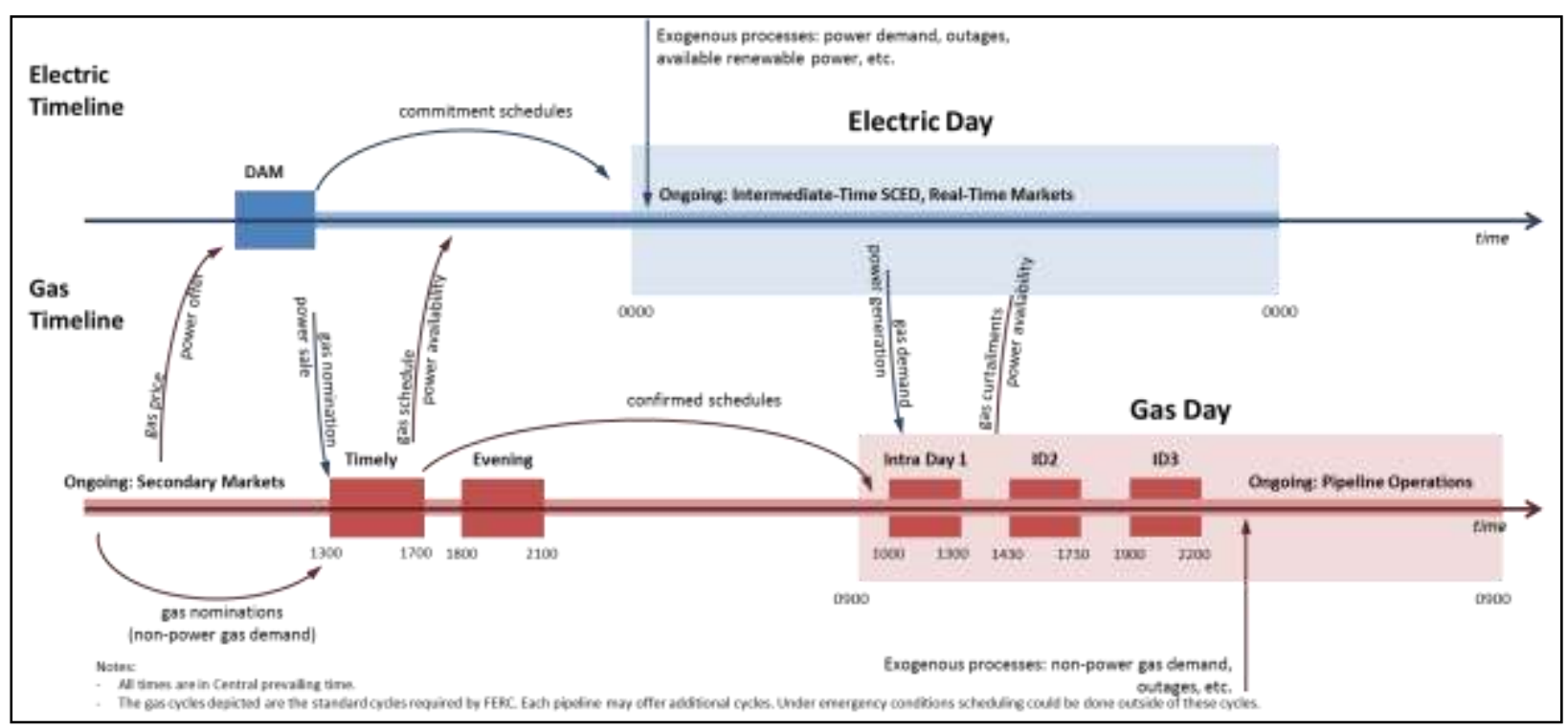

Figure 1. Gas-Electric Decision Cycles

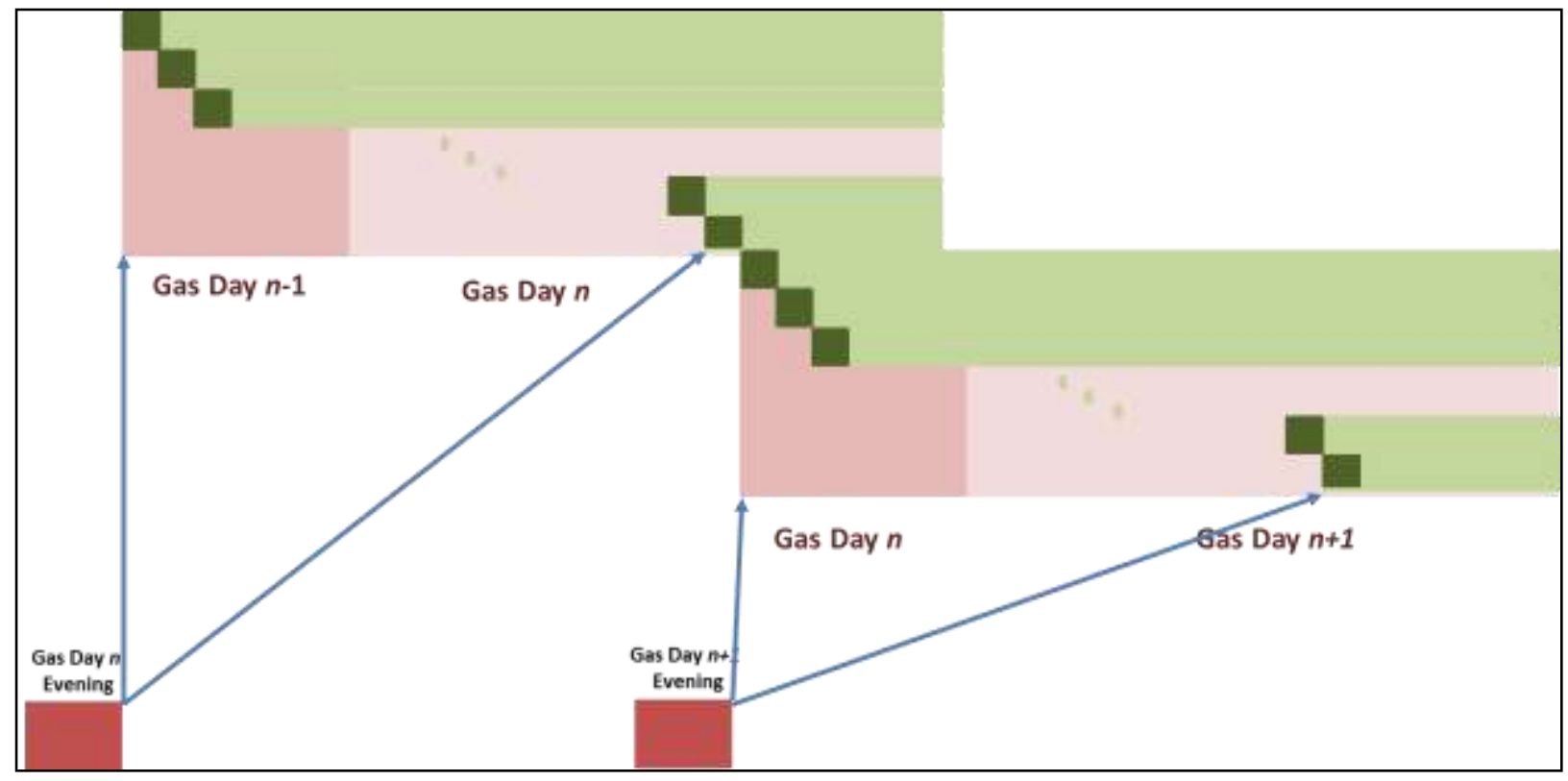

Figure 2. Succession of GMB clearings 


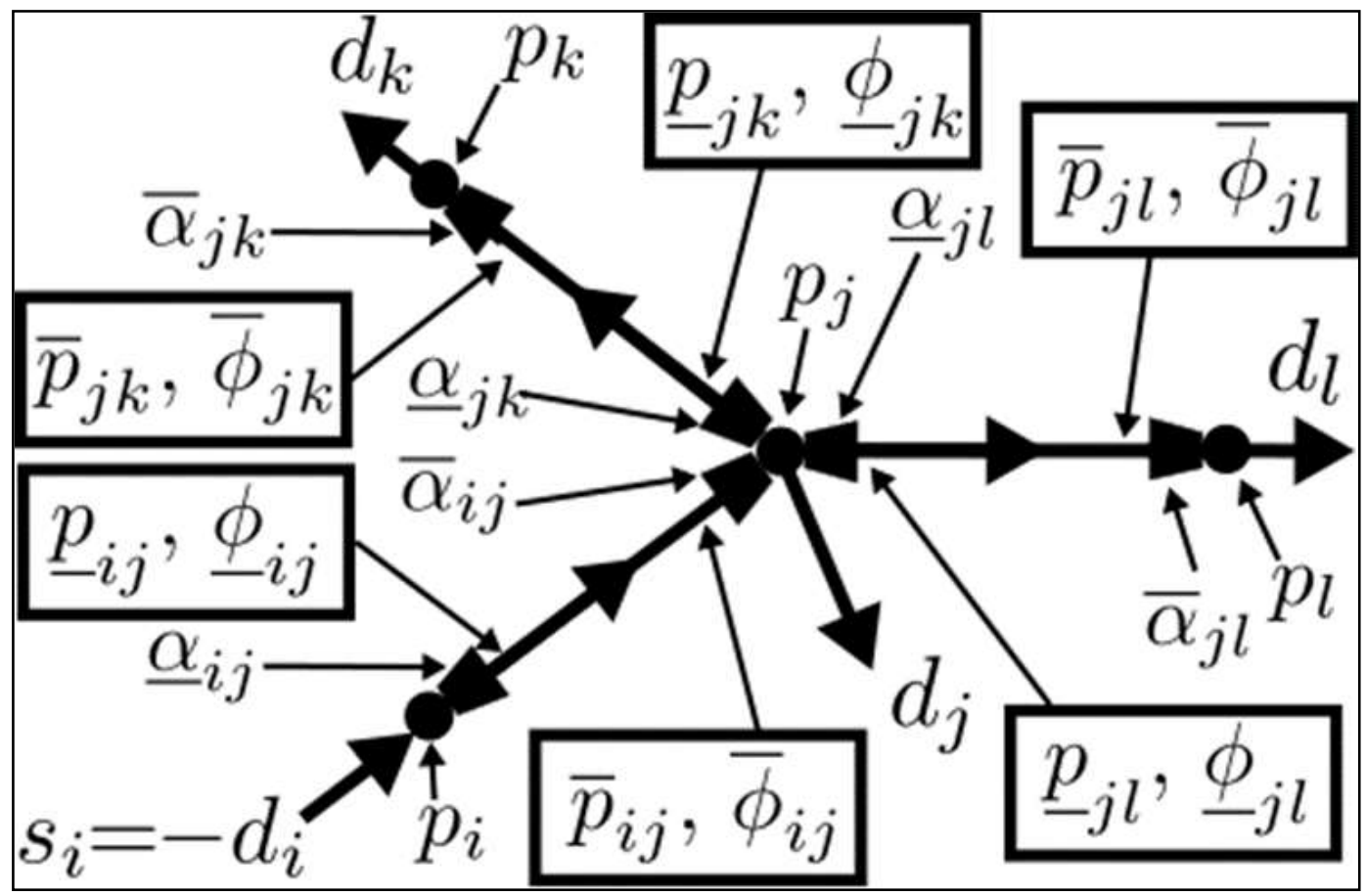

Figure 3 - Diagram of nodal control system modeling for large-scale gas transmission pipelines. Given a directed graph that represents the pipeline network, $\underline{p}_{i j}$ and $\bar{p}_{i j}$ represent pressures at the sending and receiving ends of each pipe, while $\underline{\phi}_{i j}$ and $\bar{\phi}_{i j}$ represent mass flux at the sending and receiving ends of each pipe. The quantities $\underline{\alpha}_{i j}$ and $\bar{\alpha}_{i j}$ represent pressure boost ratios of compressors that are, without loss of generality, located at every interface between a node and a pipe. Thus, nodal pressures $p_{i}$ and $p_{j}$ are related to pipe endpoint pressures $\underline{p}_{i j}$ and $\bar{p}_{i j}$ according to $\underline{p}_{i j}=\underline{\alpha}_{i j} p_{i}$ and $\bar{p}_{i j}=\bar{\alpha}_{i j} p_{j}$. The withdrawal from the network at a node $j$ is denoted by $d_{j}$, which is constructed from pre-existing contracts $\bar{q}_{j}(t)$ and secondary supply and demand profiles $\hat{s}_{j}(t)$ and $\hat{d}_{j}(t)$, or the supply injected at a node $i$ is denoted by $s_{j}$. 